\title{
A study on pointwise approximation by double singular integral operators
}

\author{
Gumrah Uysal ${ }^{* *}$, Mine Menekse Yilmaz ${ }^{2}$ and Ertan Ibikli ${ }^{3}$ \\ Dedicated to the memory of Prof. Akif Gadjiev
}

"Correspondence:

guysal@karabuk.edu.tr

'Department of Mathematics,

Faculty of Science, Karabuk

University, Karabuk, Turkey

Full list of author information is

available at the end of the article

\begin{abstract}
In the present work we prove the pointwise convergence and the rate of pointwise convergence for a family of singular integral operators with radial kernel in two-dimensional setting in the following form: $L_{\lambda}(f ; x, y)=\iint_{D} f(t, s) H_{\lambda}(t-x, s-y) d t d s$, $(x, y) \in D$, where $D=\langle a, b\rangle \times\langle c, d\rangle$ is an arbitrary closed, semi-closed or open region in $\mathbb{R}^{2}$ and $\lambda \in \Lambda, \Lambda$ is a set of non-negative numbers with accumulation point $\lambda_{0}$. Also we provide an example to justify the theoretical results.
\end{abstract}

MSC: Primary 41A35; secondary 41A25

Keywords: $\mu$-generalized Lebesgue point; radial kernel; rate of convergence; bimonotonicity; bounded bivariation

\section{Introduction}

Taberski [1] analyzed both the pointwise convergence of functions in $L_{1}(-\pi, \pi)$, where $L_{1}(-\pi, \pi)$ is the collection of all measurable functions $f$ for which $|f|$ is integrable on $(-\pi, \pi)$ and the approximation properties of their derivatives by a two parameter family of convolution type singular integral operators $U_{\lambda}(f ; x)$ of the form

$$
U_{\lambda}(f ; x)=\int_{-\pi}^{\pi} f(t) K_{\lambda}(t-x) d t, \quad x \in(-\pi, \pi) .
$$

Here, $K_{\lambda}(t)$ denotes a kernel fulfilling appropriate conditions with $\lambda \in \Lambda$, where $\Lambda$ is a given set of non-negative numbers with accumulation point $\lambda_{0}$. Following this work, Gadjiev [2] proved the pointwise convergence of operators of type (1.1) at a generalized Lebesgue point and established the pertinent convergence order. Rydzewska [3] extended these results to approximation at a $\mu$-generalized Lebesgue point. Karsli and Ibikli $[4,5]$ proceeded to the study of the more general integral operators defined by

$$
T_{\lambda}(f ; x)=\int_{a}^{b} f(t) K_{\lambda}(t-x) d t, \quad x \in\langle a, b\rangle, \lambda \in \Lambda \in \mathbb{R},
$$

with functions in $L_{1}\langle a, b\rangle$ where $\langle a, b\rangle$ is an arbitrary interval in $\mathbb{R}$ such as $[a, b],(a, b),[a, b)$ or $(a, b]$.

The convergence of the other operators have been studied at characteristic points such as a generalized Lebesgue point, $m$-Lebesgue point, and so on, by other workers: a family

() 2015 Uysal et al.: licensee Springer. This is an Open Access article distributed under the terms of the Creative Commons Attribution License (http://creativecommons.org/licenses/by/4.0), which permits unrestricted use, distribution, and reproduction in any medium, provided the original work is properly credited. 
of nonlinear singular integral operators [6,7], a family of nonlinear $m$-singular integral operators [8], Fejer-Type singular integrals [9], moment type operators [10], a family of nonlinear Mellin type convolution operators [11], nonlinear integral operators with homogeneous kernels [12] and a family of Mellin type nonlinear $m$-singular integral operators [13].

Taberski [14] stepped up his analysis to two-dimensional singular integrals of the form

$$
T_{\lambda}(f ; x, y)=\iint_{Q} f(t, s) K_{\lambda}(t-x, s-y) d t d s, \quad(x, y) \in Q,
$$

where $Q$ denotes a given rectangle. His findings were later used by Siudut $[15,16]$ rendering significant results. Yilmaz et al. [17] replaced $K_{\lambda}$ in (1.3) by a radial function $H_{\lambda}$ as follows:

$$
L_{\lambda}(f ; x, y)=\int_{-\pi}^{\pi} \int_{-\pi}^{\pi} f(t, s) H_{\lambda}(t-x, s-y) d t d s, \quad(x, y) \in\langle-\pi, \pi\rangle \times\langle-\pi, \pi\rangle .
$$

The new operator approaches $f\left(x_{0}, y_{0}\right)$ as $(x, y, \lambda)$ tends to $\left(x_{0}, y_{0}, \lambda_{0}\right)$. In [18], the function $f \in L_{1}(\langle-\pi, \pi\rangle \times\langle-\pi, \pi\rangle)$ became $f \in L_{p}(D)$ where $D=\langle a, b\rangle \times\langle c, d\rangle$ is an arbitrary closed, semi-closed or open region in $\mathbb{R}^{2}$.

The current manuscript presents a continuation and further generalization of [18]. The main purpose is to investigate the pointwise convergence and the rate of convergence of the operators in the following form:

$$
L_{\lambda}(f ; x, y)=\iint_{D} f(t, s) H_{\lambda}(t-x, s-y) d s d t, \quad(x, y) \in D,
$$

where $D=\langle a, b\rangle \times\langle c, d\rangle$ is an arbitrary closed, semi-closed or open region in $\mathbb{R}^{2}$, at a $\mu$ generalized Lebesgue point of $f \in L_{1}(D)$ as $(x, y, \lambda) \rightarrow\left(x_{0}, y_{0}, \lambda_{0}\right)$. Here $L_{1}(D)$ is the collection of all measurable functions $f$ for which $|f|$ is integrable on $D$ and the kernel function $H_{\lambda}(s, t)$ is a radial function. As concerns the study of linear singular operators in several settings, the reader may see also e.g. [19-23].

The paper is organized as follows: In Section 2, we introduce the fundamental definitions. In Section 3, we give a theorem concerning the existence of the operator of type (1.5). In Section 4, we prove two theorems about the pointwise convergence of $L_{\lambda}(f ; x, y)$ to $f\left(x_{0}, y_{0}\right)$ whenever $\left(x_{0}, y_{0}\right)$ is a $\mu$-generalized Lebesgue point of $f$ in bounded region and unbounded region. In Section 5, we establish the rate of convergence of operators of type $(1.5)$ to $f\left(x_{0}, y_{0}\right)$ as $(x, y, \lambda)$ tends to $\left(x_{0}, y_{0}, \lambda_{0}\right)$ and the paper is ended with an example to support our results.

\section{Preliminaries}

In this section we introduce the main definitions used in this paper.

Definition 1 A function $H \in L_{1}\left(\mathbb{R}^{2}\right)$ is said to be radial, if there exists a function $K: \mathbb{R}_{0}^{+} \rightarrow$ $\mathbb{R}$ such that $H(t, s)=K\left(\sqrt{t^{2}+s^{2}}\right)$ a.e. [24].

Definition 2 A point $\left(x_{0}, y_{0}\right) \in D$ is called a $\mu$-generalized Lebesgue point of function $f \in L_{1}(D)$ if

$$
\lim _{(h, k) \rightarrow(0,0)} \frac{1}{\mu_{1}(h) \mu_{2}(k)} \int_{0}^{h} \int_{0}^{k}\left|f\left(t+x_{0}, s+y_{0}\right)-f\left(x_{0}, y_{0}\right)\right| d t d s=0,
$$


where $\mu_{1}(t): \mathbb{R} \rightarrow \mathbb{R}$, absolutely continuous on $\left[-\delta_{0}, \delta_{0}\right]$, increasing on $\left[0, \delta_{0}\right]$ and $\mu_{1}(0)=$ 0 and also $\mu_{2}(s): \mathbb{R} \rightarrow \mathbb{R}$, absolutely continuous on $\left[-\delta_{0}, \delta_{0}\right]$, increasing on $\left[0, \delta_{0}\right]$ and $\mu_{2}(0)=0$. Here $0<h, k<\delta_{0}[25]$.

The following two examples are simple applications to a generalized Lebesgue point and $\mu$-generalized Lebesgue point of some functions that belong to $L_{1}\left(\mathbb{R}^{2}\right)$.

Example 1 Let $g: \mathbb{R}^{2} \rightarrow \mathbb{R}$ be given by

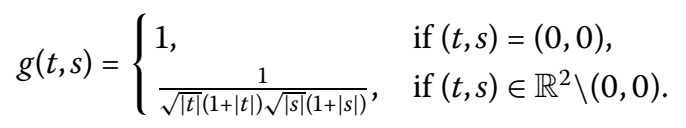

Now, if $\mu_{1}(t)=t^{\frac{1}{4}} e^{t}$ and $\mu_{2}(s)=s^{\frac{1}{4}} e^{s}$, then the origin is a $\mu$-generalized Lebesgue point of $g \in L_{1}\left(\mathbb{R}^{2}\right)$ but not a generalized Lebesgue point.

Example 2 Let $f: \mathbb{R}^{2} \rightarrow \mathbb{R}$ be given by

$$
f(t, s)= \begin{cases}e^{-(t+s)}, & \text { if }(t, s) \in(0,1] \times(0,1] \\ 0, & \text { if }(t, s) \in \mathbb{R}^{2} \backslash(0,1] \times(0,1]\end{cases}
$$

If we take $\mu_{1}(t)=t^{\frac{1}{4}+1}$ and $\mu_{2}(s)=s^{\frac{1}{4}+1}$, then the origin is a $\mu$-generalized Lebesgue point of $f \in L_{1}\left(\mathbb{R}^{2}\right)$. On the other hand, if we take $\alpha=\frac{1}{4}$ and $p=1$, then the origin is also a generalized Lebesgue point. Clearly, this example shows that generalized Lebesgue points are also $\mu$-generalized Lebesgue points.

Definition 3 (Class $A$ ) Let $H_{\lambda}: \mathbb{R}^{2} \times \Lambda \rightarrow \mathbb{R}$ be a radial function i.e., there exists a function $K_{\lambda}: \mathbb{R}_{0}^{+} \times \Lambda \rightarrow \mathbb{R}$ such that the following equality holds for $(t, s) \in \mathbb{R}^{2}$ a.e.:

$$
H_{\lambda}(t, s):=K_{\lambda}\left(\sqrt{t^{2}+s^{2}}\right)
$$

where $\Lambda$ is a given set of non-negative numbers with accumulation point $\lambda_{0}$.

$H_{\lambda}(t, s)$ belongs to class $A$, if the following conditions are satisfied:

(a) $H_{\lambda}(t, s)=K_{\lambda}\left(\sqrt{t^{2}+s^{2}}\right)$ is even, non-negative and integrable as a function of $(s, t)$ on $\mathbb{R}^{2}$ for each fixed $\lambda \in \Lambda$.

(b) For fixed $\left(x_{0}, y_{0}\right) \in D, K_{\lambda}\left(\sqrt{x_{0}^{2}+y_{0}^{2}}\right)$ tends to infinity as $\lambda$ tends to $\lambda_{0}$.

(c) $\lim _{(x, y, \lambda) \rightarrow\left(x_{0}, y_{0}, \lambda_{0}\right)} \iint_{\mathbb{R}^{2}} K_{\lambda}\left(\sqrt{(t-x)^{2}+(s-y)^{2}}\right) d t d s=1$.

(d) $\lim _{\lambda \rightarrow \lambda_{0}}\left[\sup _{\xi \leq \sqrt{t^{2}+s^{2}}} K_{\lambda}\left(\sqrt{t^{2}+s^{2}}\right)\right]=0, \forall \xi>0$.

(e) $\lim _{\lambda \rightarrow \lambda_{0}} \iint_{\xi \leq \sqrt{t^{2}+s^{2}}} K_{\lambda}\left(\sqrt{t^{2}+s^{2}}\right) d t d s=0, \forall \xi>0$.

(f) $K_{\lambda}\left(\sqrt{t^{2}+s^{2}}\right)$ is monotonically increasing with respect to $t$ on $(-\infty, 0]$ and similarly $K_{\lambda}\left(\sqrt{t^{2}+s^{2}}\right)$ is monotonically increasing with respect to $s$ on $(-\infty, 0]$ for any $\lambda \in \Lambda$. Analogously, $K_{\lambda}\left(\sqrt{t^{2}+s^{2}}\right)$ is bimonotonically increasing with respect to $(t, s)$ on $[0, \infty) \times[0, \infty)$ and $(-\infty, 0] \times(-\infty, 0]$ and bimonotonically decreasing with respect to $(t, s)$ on $[0, \infty) \times(-\infty, 0]$ and $(-\infty, 0] \times[0, \infty)$ for any $\lambda \in \Lambda$.

Throughout this paper we assume that the kernel $H_{\lambda}(t, s)$ belongs to class $A$. 


\section{Existence of the operator}

Lemma 1 If $\in L_{1}(D)$, then the operator $L_{\lambda}(f ; x, y)$ defines a continuous transformation over $L_{1}(D)[26]$.

\section{Pointwise convergence}

The following theorem gives a pointwise approximation of the integral operators of type (1.5) to the function $f$ at a $\mu$-generalized Lebesgue point of $f \in L_{1}(D)$ where $D=\langle a, b\rangle \times$ $\langle c, d\rangle$ is a bounded region in $\mathbb{R}^{2}$, which is closed, semi-closed or open.

Theorem 1 If $\left(x_{0}, y_{0}\right)$ is a $\mu$-generalized Lebesgue point of $f \in L_{1}(D)$, then

$$
\lim _{(x, y, \lambda) \rightarrow\left(x_{0}, y_{0}, \lambda_{0}\right)} L_{\lambda}(f ; x, y)=f\left(x_{0}, y_{0}\right)
$$

on any set $Z$ on which the functions

$$
\int_{x_{0}-\delta}^{x_{0}+\delta} \int_{y_{0}-\delta}^{y_{0}+\delta} K_{\lambda}\left(\sqrt{(t-x)^{2}+(s-y)^{2}}\right)\left|\left\{\mu_{1}\left(\left|t-x_{0}\right|\right)\right\}_{t}^{\prime}\right|\left|\left\{\mu_{2}\left(\left|s-y_{0}\right|\right)\right\}_{s}^{\prime}\right| d t d s
$$

and

$$
K_{\lambda}(0) \mu_{1}\left(\left|x-x_{0}\right|\right) \text { and } K_{\lambda}(0) \mu_{2}\left(\left|y-y_{0}\right|\right)
$$

are bounded as $(x, y, \lambda)$ tends to $\left(x_{0}, y_{0}, \lambda_{0}\right)$.

Proof Suppose that $\left(x_{0}, y_{0}\right) \in D$ is a $\mu$-generalized Lebesgue point of $f \in L_{1}(D)$. Therefore, for all given $\varepsilon>0$, there exists $\delta>0$ such that for all $h, k$ satisfying $0<h, k \leq \delta$, the following inequality holds:

$$
\int_{x_{0}}^{x_{0}+\delta} \int_{y_{0}-\delta}^{y_{0}}\left|f(t, s)-f\left(x_{0}, y_{0}\right)\right| d t d s<\varepsilon \mu_{1}(h) \mu_{2}(k) .
$$

If we follow the same strategy as used in the proof of Theorem 4.1 in [18], then we obtain

$$
\begin{aligned}
\left|L_{\lambda}(f ; x, y)-f\left(x_{0}, y_{0}\right)\right| \leq & \iint_{D}\left|f(t, s)-f\left(x_{0}, y_{0}\right)\right| K_{\lambda}\left(\sqrt{(t-x)^{2}+(s-y)^{2}}\right) d t d s \\
& +\left|f\left(x_{0}, y_{0}\right)\right| \iint_{\mathbb{R}^{2}} K_{\lambda}\left(\sqrt{(t-x)^{2}+(s-y)^{2}}\right) d t d s-1 \mid \\
& +\left|f\left(x_{0}, y_{0}\right)\right| \iint_{\mathbb{R}^{2} \backslash D} K_{\lambda}\left(\sqrt{(t-x)^{2}+(s-y)^{2}}\right) d t d s \\
= & I_{1}+I_{2}+I_{3} .
\end{aligned}
$$

In view of conditions (c) and (d) of class $A, I_{2} \rightarrow 0$, and $I_{3} \rightarrow 0$ as $\lambda \rightarrow \lambda_{0}$, respectively,

$$
\begin{aligned}
I_{1} & =\left\{\iint_{D \backslash B_{\delta}}+\iint_{B_{\delta}}\right\}\left|f(t, s)-f\left(x_{0}, y_{0}\right)\right| K_{\lambda}\left(\sqrt{(t-x)^{2}+(s-y)^{2}}\right) d t d s \\
& =I_{11}+I_{12},
\end{aligned}
$$

where $B_{\delta}:=\left\{(s, t):\left(s-x_{0}\right)^{2}+\left(t-y_{0}\right)^{2}<\delta^{2},\left(x_{0}, y_{0}\right) \in D\right\}$. 
Since $K_{\lambda}\left(\sqrt{t^{2}+s^{2}}\right)$ is monotonically decreasing on $D \backslash B_{\delta}$, the inequality

$$
I_{11} \leq K_{\lambda}((\sqrt{2}-1) \delta / \sqrt{2})\left(\|f\|_{L_{1}(D)}+\left|f\left(x_{0}, y_{0}\right)\right||b-a||d-c|\right)
$$

holds. Hence by condition (d) of class $A, I_{11} \rightarrow 0$ as $(x, y, \lambda) \rightarrow\left(x_{0}, y_{0}, \lambda_{0}\right)$.

Now, we prove that $I_{12}$ tends to zero as $(x, y, \lambda)$ tends to $\left(x_{0}, y_{0}, \lambda_{0}\right)$. It is easy to see that the following inequality holds for $I_{12}$, i.e.:

$$
\begin{aligned}
I_{12} \leq & \left\{\int_{x_{0}}^{x_{0}+\delta} \int_{y_{0}-\delta}^{y_{0}}+\int_{x_{0}-\delta}^{x_{0}} \int_{y_{0}-\delta}^{y_{0}}\right\}\left|f(t, s)-f\left(x_{0}, y_{0}\right)\right| K_{\lambda}\left(\sqrt{(t-x)^{2}+(s-y)^{2}}\right) d t d s \\
& +\left\{\int_{x_{0}-\delta}^{x_{0}} \int_{y_{0}}^{y_{0}+\delta}+\int_{x_{0}}^{x_{0}+\delta} \int_{y_{0}}^{y_{0}+\delta}\right\}\left|f(t, s)-f\left(x_{0}, y_{0}\right)\right| K_{\lambda}\left(\sqrt{(t-x)^{2}+(s-y)^{2}}\right) d t d s \\
= & I_{121}+I_{122}+I_{123}+I_{124} .
\end{aligned}
$$

Let us consider the integral $I_{121}$. In view of (4.3), for every $\varepsilon>0$ there exists $\delta>0$ such that

$$
\int_{x_{0}}^{x_{0}+h} \int_{y_{0}-k}^{y_{0}}\left|f(t, s)-f\left(x_{0}, y_{0}\right)\right| d t d s<\varepsilon \mu_{1}(h) \mu_{2}(k)
$$

holds for all $0<h, k \leq \delta$.

Let us define a new function by

$$
F(t, s):=\int_{x_{0}}^{t} \int_{s}^{y_{0}}\left|f(u, v)-f\left(x_{0}, y_{0}\right)\right| d u d v
$$

For all $t$ and $s$ satisfying $0<t-x_{0} \leq \delta$ and $0<y_{0}-s \leq \delta$ we have

$$
|F(t, s)| \leq \varepsilon \mu_{1}\left(t-x_{0}\right) \mu_{2}\left(y_{0}-s\right) .
$$

In view of (4.4) and (4.5) and applying the method of bivariate integration by parts to $I_{121}$ (see Theorem 2.2, p.100 in [14]) we have

$$
\begin{aligned}
I_{121} \leq & \varepsilon \int_{x_{0}}^{x_{0}+\delta} \int_{y_{0}-\delta}^{y_{0}} \mu_{1}\left(t-x_{0}\right) \mu_{2}\left(y_{0}-s\right)\left|d K_{\lambda}\left(\sqrt{(t-x)^{2}+(s-y)^{2}}\right)\right| \\
& +\varepsilon \mu_{2}(\delta) \int_{x_{0}}^{x_{0}+\delta} \mu_{1}\left(t-x_{0}\right)\left|d K_{\lambda}\left(\sqrt{(t-x)^{2}+\left(y_{0}-\delta-y\right)^{2}}\right)\right| \\
& +\varepsilon \mu_{1}(\delta) \int_{y_{0}-\delta}^{y_{0}} \mu_{2}\left(y_{0}-s\right)\left|d K_{\lambda}\left(\sqrt{\left(x_{0}+\delta-x\right)^{2}+(s-y)^{2}}\right)\right| \\
& +\varepsilon \mu_{1}(\delta) \mu_{2}(\delta) K_{\lambda}\left(\sqrt{\left(x_{0}+\delta-x\right)^{2}+\left(y_{0}-\delta-y\right)^{2}}\right) .
\end{aligned}
$$

Let us define the variations:

$$
\begin{aligned}
& B_{1}(u, v):= \begin{cases}\bigvee_{u}^{x_{0}+\delta-x} \bigvee_{y_{0}-\delta-y}^{v}\left(K_{\lambda}\left(\sqrt{t^{2}+s^{2}}\right)\right), & x_{0}-x \leq u<x_{0}+\delta-x, \\
0, & y_{0}-\delta-y<v \leq y_{0}-y,\end{cases} \\
& B_{2}(u):= \begin{cases}\bigvee_{u}^{x_{0}+\delta-x}\left(K_{\lambda}\left(\sqrt{t^{2}+\left(y_{0}-\delta-y\right)^{2}}\right)\right), & x_{0}-x \leq u<x_{0}+\delta-x, \\
0, & \text { otherwise }\end{cases}
\end{aligned}
$$




$$
B_{3}(v):= \begin{cases}\bigvee_{y_{0}-\delta-y}^{v}\left(K_{\lambda}\left(\sqrt{\left(x_{0}-x+\delta\right)^{2}+s^{2}}\right)\right), & y_{0}-\delta-y<v \leq y_{0}-y \\ 0, & \text { otherwise }\end{cases}
$$

Taking the above variations into account and applying the method of bivariate integration by parts to the last inequality, we have

$$
\begin{aligned}
I_{121} \leq & -\varepsilon \int_{x_{0}-x}^{x_{0}-x+\delta} \int_{y_{0}-y-\delta}^{y_{0}-y}\left[B_{1}(t, s)+B_{2}(t)+B_{3}(s)+K_{\lambda}\left(\sqrt{\left(x_{0}-x+\delta\right)^{2}+\left(y_{0}-\delta-y\right)^{2}}\right)\right] \\
& \times\left\{\mu_{1}\left(t-x_{0}+x\right)\right\}_{t}^{\prime}\left\{\mu_{2}\left(y_{0}-s-y\right)\right\}_{s}^{\prime} d t d s \\
= & \varepsilon\left(i_{1}+i_{2}+i_{3}+i_{4}\right) .
\end{aligned}
$$

$\operatorname{Remark} 1$ If the function $g: \mathbb{R}^{2} \rightarrow \mathbb{R}$ is bimonotonic on $\left[\alpha_{1}, \alpha_{2}\right] \times\left[\beta_{1}, \beta_{2}\right] \subset \mathbb{R}^{2}$ then the equality given by

$$
V\left(g ;\left[\alpha_{1}, \alpha_{2}\right] \times\left[\beta_{1}, \beta_{2}\right]\right)=\bigvee_{\alpha_{1}}^{\alpha_{2}} \bigvee_{\beta_{1}}^{\beta_{2}}(g(t, s))=\left|g\left(\alpha_{1}, \beta_{1}\right)-g\left(\alpha_{1}, \beta_{2}\right)-g\left(\alpha_{2}, \beta_{1}\right)+g\left(\alpha_{2}, \beta_{2}\right)\right|
$$

holds $[14,27]$.

Splitting $i_{1}$ into two parts yields

$$
\begin{aligned}
i_{1} & =-\left\{\int_{x_{0}-x}^{x_{0}-x+\delta} \int_{0}^{y_{0}-y}+\int_{x_{0}-x}^{x_{0}-x+\delta} \int_{y_{0}-y-\delta}^{0}\right\} B_{1}(t, s)\left\{\mu_{1}\left(t-x_{0}+x\right)\right\}_{t}^{\prime}\left\{\mu_{2}\left(y_{0}-s-y\right)\right\}_{s}^{\prime} d t d s \\
& =i_{11}+i_{12} .
\end{aligned}
$$

Using Remark 1 and condition (f) of class $A$, we can write for $i_{11}$

$$
\begin{aligned}
i_{11}= & -\int_{x_{0}-x}^{x_{0}-x+\delta} \int_{0}^{y_{0}-y}\left[\left\{\bigvee_{t}^{x_{0}+\delta-x} \bigvee_{y_{0}-\delta-y}^{0}+\bigvee_{t}^{x_{0}+\delta-x} \bigvee_{0}^{s}\right\} K_{\lambda}\left(\sqrt{u^{2}+v^{2}}\right)\right] \\
& \times\left\{\mu_{1}\left(t-x_{0}+x\right)\right\}_{t}^{\prime}\left\{\mu_{2}\left(y_{0}-s-y\right)\right\}_{s}^{\prime} d t d s \\
= & \int_{x_{0}-x}^{x_{0}-x+\delta} \int_{0}^{y_{0}-y}\left(K_{\lambda}\left(\sqrt{t^{2}+\left(y_{0}-\delta-y\right)^{2}}\right)-K_{\lambda}\left(\sqrt{s^{2}+\left(x_{0}+\delta-x\right)^{2}}\right)-2 K_{\lambda}(|t|)\right. \\
& \left.+K_{\lambda}\left(\sqrt{t^{2}+s^{2}}\right)+2 K_{\lambda}\left(\left|x_{0}+\delta-x\right|\right)-K_{\lambda}\left(\sqrt{\left(y_{0}-\delta-y\right)^{2}+\left(x_{0}+\delta-x\right)^{2}}\right)\right) \\
& \times\left\{\mu_{1}\left(t-x_{0}+x\right)\right\}_{t}^{\prime}\left\{\mu_{2}\left(y_{0}-s-y\right)\right\}_{s}^{\prime} d t d s .
\end{aligned}
$$

Using the same method for $i_{12}$, we have

$$
\begin{aligned}
i_{12}= & \int_{x_{0}-x}^{x_{0}-x+\delta} \int_{y_{0}-y-\delta}^{0}\left(K_{\lambda}\left(\sqrt{t^{2}+\left(y_{0}-\delta-y\right)^{2}}\right)+K_{\lambda}\left(\sqrt{s^{2}+\left(x_{0}+\delta-x\right)^{2}}\right)\right. \\
& \left.-K_{\lambda}\left(\sqrt{t^{2}+s^{2}}\right)-K_{\lambda}\left(\sqrt{\left(y_{0}-\delta-y\right)^{2}+\left(x_{0}+\delta-x\right)^{2}}\right)\right) \\
& \times\left\{\mu_{1}\left(t-x_{0}+x\right)\right\}_{t}^{\prime}\left\{\mu_{2}\left(y_{0}-s-y\right)\right\}_{s}^{\prime} d t d s .
\end{aligned}
$$


Making similar calculations for $i_{2}$ and $i_{3}$ and collecting the obtained terms, we may write

$$
\begin{aligned}
i_{1}+i_{2}+i_{3}+i_{4}= & -\int_{x_{0}-x}^{x_{0}-x+\delta} \int_{y_{0}-y-\delta}^{0} K_{\lambda}\left(\sqrt{t^{2}+s^{2}}\right)\left\{\mu_{1}\left(t-x_{0}+x\right)\right\}_{t}^{\prime}\left\{\mu_{2}\left(y_{0}-s-y\right)\right\}_{s}^{\prime} d t d s \\
& +\int_{x_{0}-x}^{x_{0}-x+\delta} \int_{0}^{y_{0}-y}\left(K_{\lambda}\left(\sqrt{t^{2}+s^{2}}\right)-2 K_{\lambda}(|t|)\right) \\
& \times\left\{\mu_{1}\left(t-x_{0}+x\right)\right\}_{t}^{\prime}\left\{\mu_{2}\left(y_{0}-s-y\right)\right\}_{s}^{\prime} d t d s .
\end{aligned}
$$

Hence the following inequality holds for $I_{121}$ :

$$
\begin{aligned}
I_{121} \leq & \varepsilon \int_{x_{0}}^{x_{0}+\delta} \int_{y_{0}-\delta}^{y_{0}} K_{\lambda}\left(\sqrt{(t-x)^{2}+(s-y)^{2}}\right)\left|\left\{\mu_{1}\left(t-x_{0}\right)\right\}_{t}^{\prime}\right|\left|\left\{\mu_{2}\left(y_{0}-s\right)\right\}_{s}^{\prime}\right| d t d s \\
& +2 \varepsilon K_{\lambda}(0) \mu_{1}(\delta) \mu_{2}\left(\left|y_{0}-y\right|\right) .
\end{aligned}
$$

By a similar argument to the evaluation of the integral $I_{121}$, we can easily obtain the following inequalities for $I_{122}, I_{123}$, and $I_{124}$ :

$$
\begin{aligned}
I_{122} \leq & \varepsilon \int_{x_{0}-\delta}^{x_{0}} \int_{y_{0}-\delta}^{y_{0}} K_{\lambda}\left(\sqrt{(t-x)^{2}+(s-y)^{2}}\right)\left|\left\{\mu_{1}\left(x_{0}-t\right)\right\}_{t}^{\prime}\right|\left|\left\{\mu_{2}\left(y_{0}-s\right)\right\}_{s}^{\prime}\right| d t d s \\
& +2 \varepsilon K_{\lambda}(0)\left(\mu_{1}(\delta) \mu_{2}\left(\left|y_{0}-y\right|\right)+\mu_{2}(\delta) \mu_{1}\left(\left|x_{0}-x\right|\right)\right) \\
& +4 \varepsilon K_{\lambda}(0) \mu_{1}\left(\left|x_{0}-x\right|\right) \mu_{2}\left(\left|y_{0}-y\right|\right), \\
I_{123} \leq & \varepsilon \int_{x_{0}-\delta}^{x_{0}} \int_{y_{0}}^{y_{0}+\delta} K_{\lambda}\left(\sqrt{(t-x)^{2}+(s-y)^{2}}\right)\left|\left\{\mu_{1}\left(x_{0}-t\right)\right\}_{t}^{\prime}\right|\left|\left\{\mu_{2}\left(s-y_{0}\right)\right\}_{s}^{\prime}\right| d t d s \\
& +2 \varepsilon K_{\lambda}(0) \mu_{2}(\delta) \mu_{1}\left(\left|x_{0}-x\right|\right), \\
I_{124} \leq & \varepsilon \int_{x_{0}}^{x_{0}+\delta} \int_{y_{0}}^{y_{0}+\delta} K_{\lambda}\left(\sqrt{(t-x)^{2}+(s-y)^{2}}\right)\left|\left\{\mu_{1}\left(t-x_{0}\right)\right\}_{t}^{\prime}\right|\left|\left\{\mu_{2}\left(s-y_{0}\right)\right\}_{s}^{\prime}\right| d t d s .
\end{aligned}
$$

Hence the following inequality is obtained for $I_{12}$ i.e.:

$$
\begin{aligned}
I_{12} \leq & \varepsilon \int_{x_{0}-\delta}^{x_{0}+\delta} \int_{y_{0}-\delta}^{y_{0}+\delta} K_{\lambda}\left(\sqrt{(t-x)^{2}+(s-y)^{2}}\right)\left|\left\{\mu_{1}\left(\left|x_{0}-t\right|\right)\right\}_{t}^{\prime}\right|\left|\left\{\mu_{2}\left(\left|y_{0}-s\right|\right)\right\}_{s}^{\prime}\right| d t d s \\
& +4 \varepsilon K_{\lambda}(0)\left(\mu_{1}(\delta) \mu_{2}\left(\left|y_{0}-y\right|\right)+\mu_{2}(\delta) \mu_{1}\left(\left|x_{0}-x\right|\right)\right) \\
& +4 \varepsilon K_{\lambda}(0) \mu_{1}\left(\left|x_{0}-x\right|\right) \mu_{2}\left(\left|y_{0}-y\right|\right) .
\end{aligned}
$$

The remaining part of the proof is obvious by the hypotheses (4.1) and (4.2). Hence $I_{12} \rightarrow 0$ as $\lambda \rightarrow \lambda_{0}$. Thus the proof is completed.

The following theorem gives a pointwise approximation of the integral operators of type (1.5) to the function $f$ at a $\mu$-generalized Lebesgue point of $f \in L_{1}\left(\mathbb{R}^{2}\right)$.

Theorem 2 Suppose that the hypothesis of Theorem 1 is satisfied for $D=\mathbb{R}^{2}$. If $\left(x_{0}, y_{0}\right)$ is a $\mu$-generalized Lebesgue point of $f \in L_{1}\left(\mathbb{R}^{2}\right)$ then

$$
\lim _{(x, y, \lambda) \rightarrow\left(x_{0}, y_{0}, \lambda_{0}\right)} L_{\lambda}(f ; x, y)=f\left(x_{0}, y_{0}\right) .
$$


Proof The proof of this theorem is quite similar to the proof of Theorem 4.2 in [18] and thus is omitted.

\section{Rate of convergence}

In this section, we give a theorem concerning the rate of pointwise convergence.

Theorem 3 Suppose that the hypotheses of Theorem 1 and Theorem 2 are satisfied. Let

$$
\begin{aligned}
\Delta(\lambda, \delta, x, y)= & \int_{x_{0}-\delta}^{x_{0}+\delta} \int_{y_{0}-\delta}^{y_{0}+\delta} K_{\lambda}\left(\sqrt{(t-x)^{2}+(s-y)^{2}}\right) \\
& \times\left|\left\{\mu_{1}\left(\left|t-x_{0}\right|\right)\right\}_{t}^{\prime}\right|\left|\left\{\mu_{2}\left(\left|s-y_{0}\right|\right)\right\}_{s}^{\prime}\right| d t d s
\end{aligned}
$$

for $\delta>0$ and the following assumptions be satisfied:

(i) $\Delta(\lambda, \delta, x, y) \rightarrow 0$ as $(x, y, \lambda) \rightarrow\left(x_{0}, y_{0}, \lambda_{0}\right)$ for some $\delta>0$.

(ii) For every $\xi>0$

$$
\begin{aligned}
K_{\lambda}(\xi) & =o(\Delta(\lambda, \delta, x, y)) \\
\text { as }(x, y, \lambda) & \rightarrow\left(x_{0}, y_{0}, \lambda_{0}\right) .
\end{aligned}
$$

(iii) For every $\xi>0$

$$
\begin{aligned}
& \iint_{\xi \leq \sqrt{s^{2}+t^{2}}} K_{\lambda}\left(\sqrt{t^{2}+s^{2}}\right) d t d s=o(\Delta(\lambda, \delta, x, y)) \\
& \text { as }(x, y, \lambda) \rightarrow\left(x_{0}, y_{0}, \lambda_{0}\right) .
\end{aligned}
$$

Then at each $\mu$-generalized Lebesgue point of $f \in L_{1}\left(\mathbb{R}^{2}\right)$ we have as $(x, y, \lambda) \rightarrow\left(x_{0}, y_{0}, \lambda_{0}\right)$

$$
\left|L_{\lambda}(f ; x, y)-f\left(x_{0}, y_{0}\right)\right|=o(\Delta(\lambda, \delta, x, y)) .
$$

Proof Under the hypotheses of Theorem 1 and Theorem 2 we can write

$$
\begin{aligned}
& \left|L_{\lambda}(f ; x, y)-f\left(x_{0}, y_{0}\right)\right| \\
& \leq \varepsilon \int_{x_{0}-\delta}^{x_{0}+\delta} \int_{y_{0}-\delta}^{y_{0}+\delta} K_{\lambda}\left(\sqrt{(t-x)^{2}+(s-y)^{2}}\right)\left|\left\{\mu_{1}\left(\left|x_{0}-t\right|\right)\right\}_{t}^{\prime}\right|\left|\left\{\mu_{2}\left(\left|y_{0}-s\right|\right)\right\}_{s}^{\prime}\right| d t d s \\
& \quad+4 \varepsilon K_{\lambda}(0)\left(\mu_{1}(\delta) \mu_{2}\left(\left|y_{0}-y\right|\right)+\mu_{2}(\delta) \mu_{1}\left(\left|x_{0}-x\right|\right)\right) \\
& \quad+4 \varepsilon K_{\lambda}(0) \mu_{1}\left(\left|x_{0}-x\right|\right) \mu_{2}\left(\left|y_{0}-y\right|\right) \\
& \quad+K_{\lambda}((\sqrt{2}-1) \delta / \sqrt{2})\|f\|_{L_{1}\left(\mathbb{R}^{2}\right)}+\left|f\left(x_{0}, y_{0}\right)\right| \iint_{(\sqrt{2}-1) \delta / \sqrt{2} \leq \sqrt{s^{2}+t^{2}}} K_{\lambda}\left(\sqrt{t^{2}+s^{2}}\right) d t d s \\
& \quad+\left|f\left(x_{0}, y_{0}\right)\right|\left|\iint_{\mathbb{R}^{2}} K_{\lambda}\left(\sqrt{(t-x)^{2}+(s-y)^{2}}\right) d t d s-1\right| .
\end{aligned}
$$

From (i)-(iii) and using conditions of class $A$, we have the desired result i.e.,

$$
\left|L_{\lambda}(f ; x, y)-f\left(x_{0}, y_{0}\right)\right|=o(\Delta(\lambda, \delta, x, y)) .
$$


Example 3 Let $\Lambda=(0, \infty), \lambda_{0}=0$, and

$$
H_{\lambda}(t, s)=\frac{1}{4 \pi \lambda} e^{\frac{-\left(t^{2}+s^{2}\right)}{4 \lambda}} .
$$

To verify that $H_{\lambda}(t, s)$ satisfies the hypotheses of Theorem 1 and Theorem 2 see [15].

Let $\left(x_{0}, y_{0}\right)=(0,0), \mu_{1}(t)=t$ and $\mu_{2}(s)=s$. Hence we obtain

$$
\begin{aligned}
\Delta(\lambda, \delta, x, y) & =\int_{-\delta}^{+\delta} \int_{-\delta}^{+\delta} \frac{1}{4 \pi \lambda} e^{\frac{-\left((t-x)^{2}+(s-y)^{2}\right)}{4 \lambda}} d t d s \\
& =\frac{1}{2}\left(\operatorname{Erf}\left(\frac{\delta-x}{2 \sqrt{\lambda}}\right)+\operatorname{Erf}\left(\frac{x}{2 \sqrt{\lambda}}\right)\right)\left(\operatorname{Erf}\left(\frac{\delta-y}{2 \sqrt{\lambda}}\right)+\operatorname{Erf}\left(\frac{y}{2 \sqrt{\lambda}}\right)\right) .
\end{aligned}
$$

In order to find for which $\delta>0$ the condition (i) in Theorem 3 is satisfied, let $\Delta(\lambda, \delta, x, y) \rightarrow$ 0 as $(x, y, \lambda) \rightarrow(0,0,0)$. Hence

$$
\lim _{(x, y, \lambda) \rightarrow(0,0,0)} \Delta(\lambda, \delta, x, y)=0
$$

if and only if $\delta^{2}=o(\lambda)$. Consequently, the following equality holds:

$$
\Delta(\lambda, \delta, x, y)=O(\lambda)
$$

Finally, in order to get finite limit values from the expressions

$$
\begin{aligned}
\lim _{(x, y, \lambda) \rightarrow\left(x_{0}, y_{0}, \lambda_{0}\right)} K_{\lambda}(0) \mu_{1}\left(\left|x_{0}-x\right|\right) & =\lim _{(x, y, \lambda) \rightarrow(0,0,0)} \frac{1}{4 \pi \lambda} e^{\frac{-\left(x^{2}+y^{2}\right)}{4 \lambda}}|x|, \\
\lim _{(x, y, \lambda) \rightarrow\left(x_{0}, y_{0}, \lambda_{0}\right)} K_{\lambda}(0) \mu_{2}\left(\left|y_{0}-y\right|\right) & =\lim _{(x, y, \lambda) \rightarrow(0,0,0)} \frac{1}{4 \pi \lambda} e^{\frac{-\left(x^{2}+y^{2}\right)}{4 \lambda}}|y|,
\end{aligned}
$$

the rates of convergence $\frac{1}{4 \pi \lambda} e^{\frac{-\left(x^{2}+y^{2}\right)}{4 \lambda}} \rightarrow \infty$ and $|x| \rightarrow 0$ and also $\frac{1}{4 \pi \lambda} e^{\frac{-\left(x^{2}+y^{2}\right)}{4 \lambda}} \rightarrow \infty$ and $|y| \rightarrow 0$ must be equivalent. Note that $|x|=|y|=O(\lambda)$.

Hence

$$
\left|L_{\lambda}(f ; x, y)-f\left(x_{0}, y_{0}\right)\right|=o(\Delta(\lambda, \delta, x, y))=o(\lambda) .
$$

\section{Competing interests}

The authors declare that they have no competing interests.

\section{Authors' contributions}

All authors contributed equally to the writing of this paper. All authors read and approved the final manuscript.

\section{Author details}

'Department of Mathematics, Faculty of Science, Karabuk University, Karabuk, Turkey. ${ }^{2}$ Department of Mathematics, Faculty of Arts and Science, Gaziantep University, Gaziantep, Turkey. ${ }^{3}$ Department of Mathematics, Faculty of Science, Ankara University, Tandogan, Ankara, Turkey.

\section{Acknowledgements}

The authors thank the referees for their valuable comments and suggestions for the improvement of the manuscript. 


\section{References}

1. Taberski, R: Singular integrals depending on two parameters. Rocznicki Polskiego towarzystwa matematycznego, Seria I. Prace matematyczne, VII (1962)

2. Gadjiev, AD: The order of convergence of singular integrals which depend on two parameters. In: Special Problems of Functional Analysis and Their Applications to the Theory of Differential Equations and the Theory of Functions, pp. 40-44. Izdat. Akad. Nauk Azerbaĭdžan. SSR. Baku (1968)

3. Rydzewska, B: Approximation des fonctions par des intégrales singulières ordinaires. Fasc. Math. 7, 71-81 (1973)

4. Karsli, $\mathrm{H}$, Ibikli, E: Approximation properties of convolution type singular integral operators depending on two parameters and of their derivatives in $L_{1}(a, b)$. In: Proc. 16th Int. Conf. Jangjeon Math. Soc., vol. 16, pp. 66-76 (2005)

5. Karsli, H, Ibikli, E: On convergence of convolution type singular integral operators depending on two parameters. Fasc. Math. 38, 25-39 (2007)

6. Karsli, $\mathrm{H}$ : Convergence and rate of convergence by nonlinear singular integral operators depending on two parameters. Appl. Anal. 85(6-7), 781-791 (2006)

7. Karsli, $\mathrm{H}$ : On the approximation properties of a class of convolution type nonlinear singular integral operators Georgian Math. J. 15(1), 77-86 (2008)

8. Karsli, H: Fatou type convergence of nonlinear $m$-singular integral operators. Appl. Math. Comput. 246, 221-228 (2014)

9. Bardaro, C, Gori Cocchieri, C: On the degree of approximation for a class of singular integrals. Rend. Mat. 4(4), 481-490 (1984) (in Italian)

10. Bardaro, C: On approximation properties for some classes of linear operators of convolution type. Atti Semin. Mat. Fis. Univ. Modena 33(2), 329-356 (1984)

11. Bardaro, C, Mantellini, I: Pointwise convergence theorems for nonlinear Mellin convolution operators. Int. J. Pure Appl. Math. 27(4), 431-447 (2006)

12. Bardaro, C, Vinti, G, Karsli, H: Nonlinear integral operators with homogeneous kernels: pointwise approximation theorems. Appl. Anal. 90(3-4), 463-474 (2011)

13. Bardaro, C, Karsli, H, Vinti, G: On pointwise convergence of Mellin type nonlinear $m$-singular integral operators. Commun. Appl. Nonlinear Anal. 20(2), 25-39 (2013)

14. Taberski, R: On double integrals and Fourier series. Ann. Pol. Math. 15, 97-115 (1964)

15. Siudut, S: On the convergence of double singular integrals. Comment. Math. Prace Mat. 28(1), 143-146 (1988)

16. Siudut, S: A theorem of Romanovski type for double singular integrals. Comment. Math. Prace Mat. 29, 277-289 (1989)

17. Yilmaz, MM, Serenbay, SK, Ibikli, E: On singular integrals depending on three parameters. Appl. Math. Comput. 218(3), 1132-1135 (2011)

18. Yilmaz, MM, Uysal, G, Ibikli, E: A note on rate of convergence of double singular integral operators. Adv. Differ. Equ. 2014, 287 (2014)

19. Angeloni, L, Vinti, G: Convergence and rate of approximation for linear integral operators in BV- $\varphi$ spaces in multidimensional setting. J. Math. Anal. Appl. 349, 317-334 (2009)

20. Angeloni, L, Vinti, G: Approximation with respect to Goffman-Serrin variation by means of non-convolution type integral operators. Numer. Funct. Anal. Optim. 31, 519-548 (2010)

21. Bardaro, C, Karsli, H, Vinti, G: On pointwise convergence of linear integral operators with homogeneous kernel. Integral Transforms Spec. Funct. 19(6), 429-439 (2008)

22. Costarelli, D, Vinti, G: Approximation by multivariate generalized sampling Kantorovich operator in the setting of Orlicz spaces. Boll. UMl 4, 445-468 (2011)

23. Vinti, G, Zampogni, L: A unifying approach to convergence of linear sampling type operators in Orlicz spaces. Adv. Differ. Equ. 16, 573-600 (2011)

24. Bochner, S, Chandrasekharan, K: Fourier Transforms. ix+219 pp. Annals of Mathematics Studies, vol. 19. Princeton University Press, Princeton (1949)

25. Serenbay, SK, Dalmanoglu, O, Ibikli, E: On convergence of singular integral operators with radial kernels. In: Approximation Theory XIV: San Antonio 2013. Springer Proceedings in Mathematics \& Statistics, vol. 83, pp. 295-308 (2014)

26. Yilmaz, MM: On convergence of singular integral operators depending on three parameters with radial kernels. Int. J. Math. Anal. 4(39), 1923-1928 (2010)

27. Ghorpade, SR, Limaye, BV: A Course in Multivariable Calculus and Analysis. xii+475 pp. Springer, New York (2010)

\section{Submit your manuscript to a SpringerOpen ${ }^{\circ}$ journal and benefit from:}

- Convenient online submission

Rigorous peer review

- Immediate publication on acceptance

Open access: articles freely available online

- High visibility within the field

- Retaining the copyright to your article 